\title{
Direct observation of breathing phenomenon and phase transformation in Ni-rich cathode materials by in situ TEM
}

Weiqun $\mathrm{Li}^{1}$, Ioannis Siachos ${ }^{1}$, Juhan Lee ${ }^{2}$, Serena A. Corr ${ }^{3}$, Clare P. Grey ${ }^{4}$, Nigel D. Browning ${ }^{5}$ and B. Layla Mehdi ${ }^{6}$

${ }^{1}$ Department of Mechanical, Materials and Aerospace Engineering, University of Liverpool, Liverpool L69 3GH, UK, United States, ${ }^{2}$ Department of Mechanical, Materials and Aerospace Engineering, University of Liverpool, Liverpool L69 3GH, UK, Liverpool, England, United States, ${ }^{3}$ University of Sheffield, United States, ${ }^{4}$ University of Cambridge, United States, ${ }^{5}$ University of Liverpool, United States, ${ }^{6}$ Department of Mechanical, Materials and Aerospace Engineering, University of Liverpool, Liverpool L69 3GH, UK, England, United Kingdom

Nickel-rich lithium transition metal oxides are becoming the most promising cathode materials for lithium ion batteries (LIB), such as $\mathrm{LiNi}_{x} \mathrm{Mn}_{y} \mathrm{Co}_{1-x-y} \mathrm{O}_{2}$ (NMC, $\left.x \geq 0.5\right), \mathrm{LiNi}_{x} \mathrm{Co}_{y} \mathrm{Al}_{1-x-y} \mathrm{O}_{2}(\mathrm{NCA}, x \geq 0.5)$ and $\mathrm{LiNiO}_{2}$ [1][2]. The energy density of Ni-rich cathode can be enhanced by increasing the ratio of $\mathrm{Ni}$. However, the cycle life of Ni-rich cathode is reduced due to its structural degradation and thermal instability during electrochemical cycling. Therefore, the degradation mechanisms must be studied in order to provide design and manufacture strategies for $\mathrm{Ni}$-rich cathode materials that will be implemented in next generation of electric vehicles.

Currently the degradation mechanism of the layered Ni-rich cathodes e.g. LiNi0.8Mn0.1 Co0.1 $\mathrm{O}_{2}$ (NMC811), stems from the high interfacial lattice strain between the reconstructed rock-salt surface and the bulk layered structure where such surface reconstruction is surface facet-dependent [3][4]. So far the in situ TEM heating experiments showed structural and compositional evolution of Ni-rich cathode focused on the intergranular cracking along the grain boundaries in layered cathodes e.g. $\mathrm{LiNi}_{0.4} \mathrm{Mn}_{0.4} \mathrm{Co}_{0.2} \mathrm{O}_{2}$ as well as the local phase transformation induced stress buildup [5]. Moreover, the rock-salt phase was formed along the coherent twin boundary due to the Li/transition metal ion mixing in the layered phases of $\mathrm{LiNi} 0.76 \mathrm{Mn} 0.14 \mathrm{Co} 0.10 \mathrm{O}_{2}$ cathode during delithiation [6]. Additionally, dissolution process of $\mathrm{Mn}$ and Co can also be observed during galvanostatic charge/discharge cycling in in situ liquid TEM experiments [7].

In this work, we report first direct observation of breathing phenomena and phase transformation in Nirich cathode materials upon charge and discharge cycling by using in situ (S)TEM open cell approach (Figure 1a). We can observe breathing phenomena, causing volume expansion during delithiation and contraction during lithiation, as well as rapid formation of new Ni-rich phases in the Ni-rich cathode (Figure 1). We can also observe the formation of rock salt structure in NMC cathode during in situ and ex situ STEM experiments (Figure 2) as the initial layered structure (Figure 2a), and the surface area is gradually transformed into the rock salt structure during the cycling (Figures $2 b-c$ ), indicating the rapid formation of rock salt structure from the edge of the particle inwards. This mechanism is in agreement with the ex situ dark-field STEM image (Figure 2d) where phase transformation occurs at the surface of NMC cathode after cycling [3]. In both cases we can identify the core area as layered R-3m structure (Figure 2e), and the surface area as rock-salt Fm-3m (Figure 2f). This indicates that the phase 
transformation process starts from the edge and progresses inwards to the core of the particle during ling, shedding light on degradation mechanisms of the Ni-rich cathode materials for long cycle life applications.
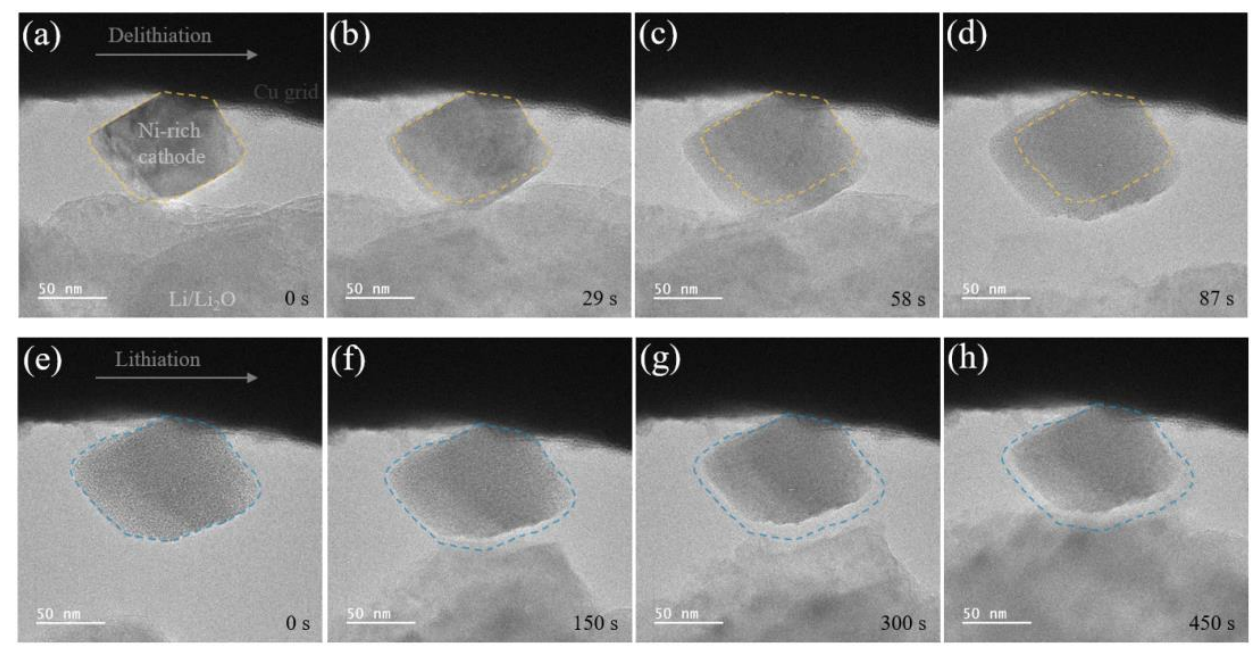

Figure 1. In situ TEM images showing the breathing phenomenon during (a-d) delithiation and (e-h) lithiation of a Ni-rich cathode nanoparticle, respectively. The orange and blue dashed lines indicate the original sizes of the Ni-rich cathode nanoparticle at the beginning of delithiation and lithiation, respectively.
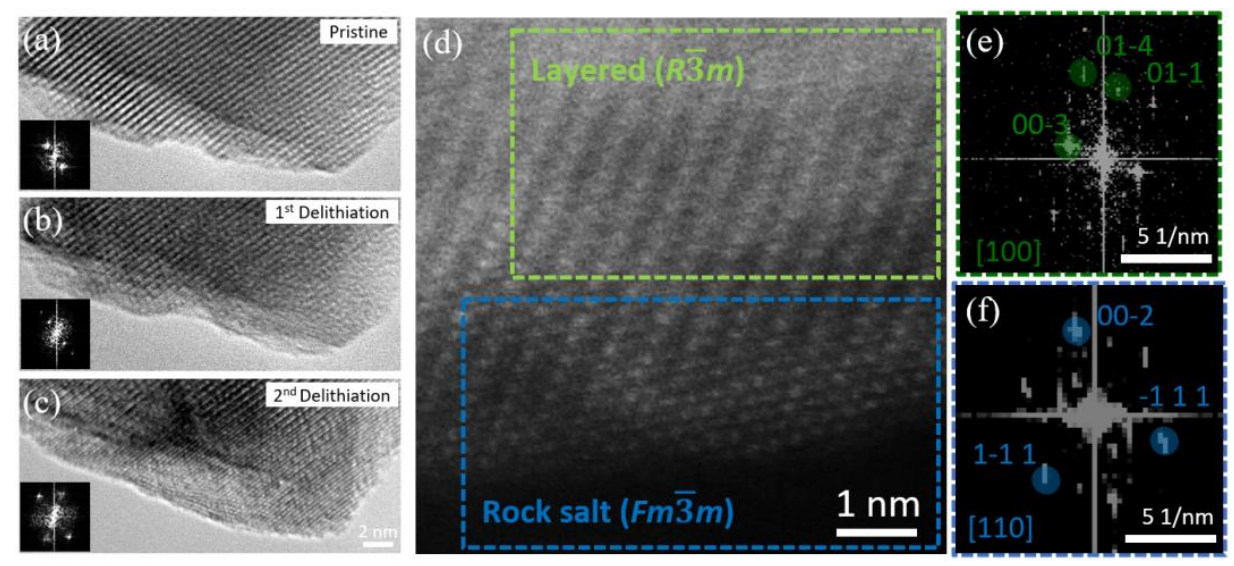

Figure 2. In situ HRTEM images of a NMC cathode nanoparticle and their corresponding FFTs (a) at the pristine state, (b) after 1st delithiation, and (c) after 2nd delithiation. (d) Ex situ dark-field STEM image of an aged NMC particle after 300 cycles. (e,f) FFTs of the corresponding regions in Figure d.

\section{References}

[1] J. Kim et al, Advanced Energy Materials 8 (2018), p. 1702028.

[2] Y. Xia et al, Nano Energy 49 (2018), p. 434.

[3] C. Xu et al, Nature Materials 20 (2020), p. 84.

[4] J. Zhu et al, ACS Applied Energy Materials 3 (2020), p. 4799.

[5] L. Mu et al, Nano Letters 18 (2018), p. 3241.

[6] S. Li et al, Angewandte Chemie 132 (2020), p. 22276.

[7] J. Hou et al, Journal of Physics: Energy 2 (2020), p. 34007.

[8] This work was supported by the EPSRC UK Faraday Institution under the Degradation (FIRG01) and Characterization (FIRG013) projects. 